\title{
Origin of the Chemiresistive Response of Ultrathin Films of Conductive Metal-Organic Frameworks
}

\author{
Víctor Rubio-Giménez, ${ }^{\dagger}$ Neyvis Almora-Barrios,${ }^{\dagger}$ Garin Escorcia-Ariza,${ }^{\dagger}$ Marta Galbiati, Michele \\ Sessolo, Sergio Tatay* and Carlos Martí-Gastaldo*
}

Instituto de Ciencia Molecular, Universitat de València, Catedrático José Beltrán 2, 46980 Paterna, Spain

\begin{abstract}
Conductive Metal-Organic Frameworks are opening new perspectives for the use of these porous materials for applications traditionally limited to more classical inorganic materials, like their integration into electronic devices. This has enabled the development of chemiresistive sensors capable of transducing the presence of specific guests into an electrical response with good selectivity and sensitivity. By combining experimental data with computational modelling, we describe a possible origin for the underlying mechanism of this phenomenon in ultrathin films $(\sim 30 \mathrm{~nm})$ of Cu-CAT-1.
\end{abstract}

The rise of electrically conductive Metal-Organic Frameworks (MOFs) has postulated these coordination frameworks, traditionally considered insulating, as promising alternatives to classical conductive materials for the development of electronic devices. ${ }^{[1]}$ The combination of high crystallinity, chemical versatility and porosity with electrical conductivity makes them appealing candidates for energy storage platforms, ${ }^{[2-4]}$ field-effect transistors (FETs) ${ }^{[5-7]}$ Schottky barrier diodes, ${ }^{[8]}$ thermoelectrics, ${ }^{[9,10]}$ resistive random-access memories, ${ }^{[11]}$ rectifiers ${ }^{[12]}$ or ion-to-electron transducers. ${ }^{[13]}$ Besides the search for new materials, research efforts have centered in gaining chemical control over their design to optimize the electrical conductivity. This can be done either intrinsically, by systematically varying the metallic cation and/or functionalizing the linker, ${ }^{[14-18]}$ or extrinsically, by using their porosity to infiltrate redox active molecules that can lead to an increase in conductivity from strong electronic coupling with the host. ${ }^{[19,20]}$ Among the conductive MOFs available, ${ }^{[21]}$ two-dimensional (2D) MOFs ${ }^{[22]}$ are specially interesting because of their high conductivity, as a result of in-plane charge delocalization and extended $\pi$-conjugation along the sheets, and the possibility to be integrated in electronic devices by using soft bottom-up methodologies. ${ }^{[5,23]}$ In these systems, single metal atoms and benzene or triphenylene linkers with $\mathrm{S}, \mathrm{N}$ or $\mathrm{O}$ as donor groups bond into $2 \mathrm{D}$ honeycomb layers that stack together to form hexagonal channels. ${ }^{[6,7,15,24-26]}$ The

[a] V. Rubio-Giménez, Dr. N. Almora-Barrios G. Escorcia-Ariza, Dr. M. Galbiati, Dr. M. Sessolo, Dr. S. Tatay, Dr. C. Martí-Gastaldo. Instituto de Ciencia Molecular

Universitat de València

Catedrático José Beltrán 2, 46980 Paterna (Spain)

e-mail: carlos.marti@uv.es, sergio.tatay@uv.es

Homepage: http://www.icmol.es/funimat

†. V.R.-G., N. A.-B and G. E.-A. contributed equally to this work.

Supporting information for this article is given via a link at the end of the document.

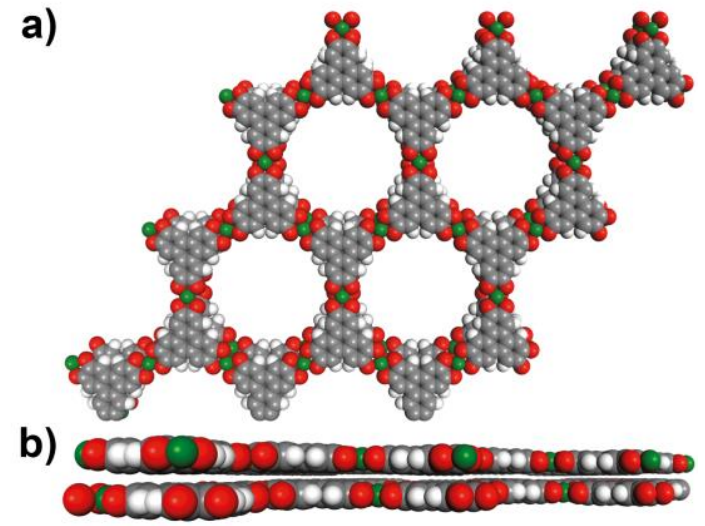

Figure 1. a) Honeycomb-like lattice and b) packing of Cu-CAT-1.

large bulk conductivity and processability of this family of $2 \mathrm{D}$ MOFs has enabled the development of solid-supported devices based on micro and nanometric thick films of $\mathrm{M}_{3}(\mathrm{HITP})_{2}$ and $\mathrm{M}_{3}(\mathrm{HHTP})_{2}(\mathrm{M}=\mathrm{Fe}$, Co, $\mathrm{Ni}$, or $\mathrm{Cu}, \mathrm{HITP}=2,3,6,7,10,11$ hexaiminotriphenylene) for selective and fast chemiresistive sensing of ammonia $\left(\mathrm{NH}_{3}\right)$ and volatile organic compounds. ${ }^{[27-29]}$ The coordination of the metal center in the network is for most cases square planar, but also octahedral for HHTP (2,3,6,7,10,11-hexahydroxytriphenylene) and $\mathrm{M}=\mathrm{Co}$ or $\mathrm{Ni},{ }^{[15]}$ which have two axial water molecules. These early works confirm a direct dependence of the electrical response with host/guest interactions that can be also modified for different metal nodes. However, further information that would help to unveil the exact mechanism controlling this phenomenon is still missing.

We recently reported a bottom-up approach to fabricate very thin $(10 \mathrm{~nm})$, highly-oriented, semiconductive $\mathrm{Cu}_{3}(\mathrm{HHTP})_{2}$ (Cu-CAT1; Figure 1) films. ${ }^{[23]}$ The sequential transfer of Cu-CAT-1 layers pre-assembled in a Langmuir-Blodgett (LB) trough over a substrate modified with a Self-Assembled Monolayer (SAM) allowed to integrate Cu-CAT-1 films into FET-type devices with high consistency and comparability. ${ }^{[30]}$ We hypothesized that these devices would be more adequate to understand more precisely the working principle behind chemiresistive response, neglecting other interfering factors that affect the conductivity of MOFs like the morphology of the sample. ${ }^{[31]}$ Through the combination of experimental data and computational modelling, we depict a possible origin for the chemiresistive response of ultrathin films (thickness $\sim 30 \mathrm{~nm}$ ) of Cu-CAT-1. Our results 
suggest that changes to the electrical conductivity are controlled by the ability of the guest to coordinate to the open metal sites in the 2D MOF layer. Molecules capable of stronger interaction like $\mathrm{NH}_{3}$ or $\mathrm{H}_{2} \mathrm{O}$ can induce a change in the coordination geometry that has a direct impact over the electronic structure of the solid. By using our method, we fabricated multiple samples by sequential transfer of Cu-CAT-1 layers on pre-patterned chips, which consisted of $500 \mu \mathrm{m}$ width interdigitated $\mathrm{Au}$ electrodes (channel length $2.5 \mu \mathrm{m}$ ) on top of a $\mathrm{Si} / \mathrm{SiO}_{2}$ substrate functionalized with OTS SAMs (OTS = octadecyl trichlorosilane). Further details about the fabrication of ultrathin films with this method are available from our previous work. ${ }^{[23]}$ We first evaluated the surface quality and thickness of the Cu-CAT-1 ultrathin films onto model $\mathrm{Si} / \mathrm{SiO}_{2} / \mathrm{OTS}$ substrates using optical microscopy scanning electron microscopy (SEM) and atomic force microscopy (AFM). All of them point to the deposition of continuous, smooth films compatible with device fabrication (Figure SI1). The thickness of a 3-transfer film, as evaluated by AFM, corresponds to an average value of $29.4 \pm 2.1 \mathrm{~nm}(10.1 \pm$ $1.1 \mathrm{~nm}$ per LB transfer cycle). Based on our previous synchrotron diffraction analysis and the proposed structural model consistent with a preferential orientation of Cu-CAT layers lying parallel to the substrate, it is safe to assume this thickness would equal to 89 monolayers considering an interlayer separation of $0.33 \mathrm{~nm}$. Surface roughness was estimated by calculating the average root-mean square (RMS) roughness over $1 \mu \mathrm{m}^{2}$ areas (Figure SI2). The obtained value of $12.9 \pm 1.0 \mathrm{~nm}$ corresponds to approximately 39 layers. To evaluate the effect of environmental changes in the conductivity of these MOF-based devices, we designed and fabricated a sealed chamber that enables a continuous read-out of the electrical response of the chips with dynamic gas changes. This setup is schematized in Figure $\mathbf{2 a} \mathbf{a} \mathbf{b}$. Here, samples could be exposed at room temperature to vacuum and different gas environments, injected into the chamber through dedicated valves.

A constant $0.1 \mathrm{~V}$ bias voltage was applied across the electrodes with a voltage source while the current flowing through the MOF film was measured using an electrometer. As highlighted above, MOFs' porosity can make their electrical conductivity extremely sensitive to changes in the environment. ${ }^{[31]}$ For that reason, we started our measurements by pumping the system to a base pressure of $10^{-6}$ mbar until a stable conductivity value was reached. Then, dynamic vacuum was removed and gas injected into the chamber. After a steady conductivity value was once again registered, we carried out the next vacuum/gas cycle. We repeated this procedure for 12 devices distributed in 3 different samples using nitrogen, oxygen, argon, synthetic air, ambient (65\% relative humidity) and $\mathrm{NH}_{3} / \mathrm{N}_{2}$ mixtures (500 ppm). Small electrical drift (Figure SI3), and ohmic contacts between the film and the electrodes (i.e. Linear I-V curves) were measured under all conditions (Figure SI4). Figure 2c shows the conductivity changes of $\sim 30 \mathrm{~nm}$ thick Cu-CAT-1 films when exposed to three consecutive vacuum $/ \mathrm{N}_{2}$ cycles. Plateau average conductivity values for this set of measures, and their standard deviations, are represented on the right side of Figure 2c. Average conductivity values measured for each tested atmosphere are reported in Figure 2d and Figure SI6. Our experimental data suggest a strong influence of the environment over the films resistance. Variation is quite homogeneous for gases without donor atoms, whereas exposure to room conditions results in very heterogeneous electrical response. This highlights the necessity of controlling the atmosphere in which the electrical conductivity a)
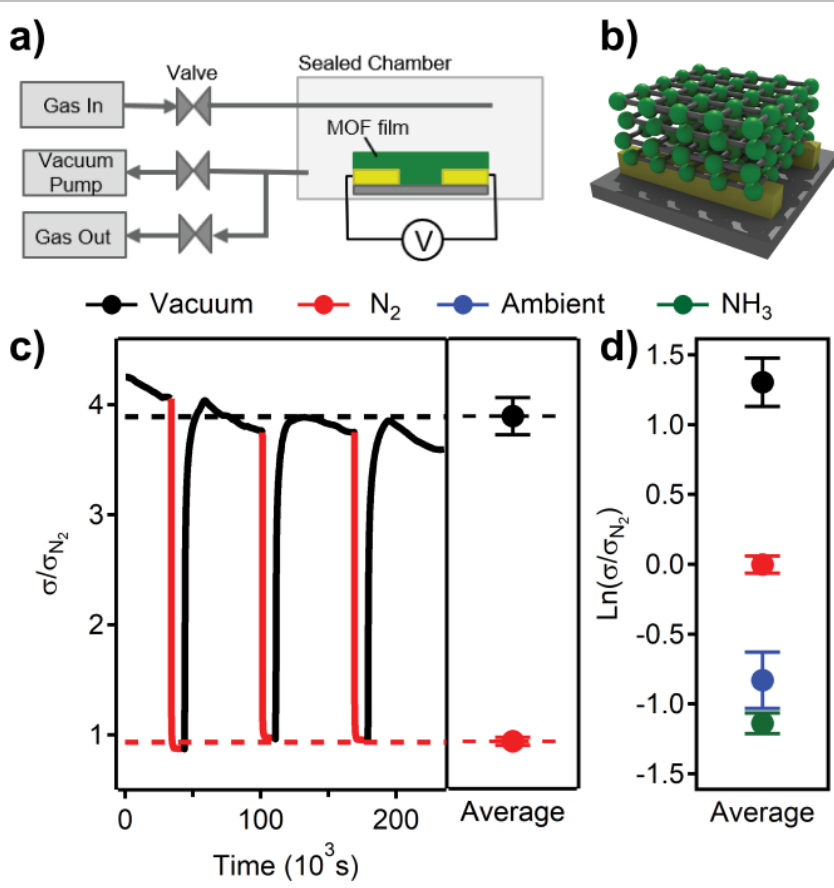

Figure 2. a) Schematic of the measurement system. b) Scheme depicting a MOF device fabricated by sequential transfer of LB-grown ultrathin films onto prepatterned Au electrodes. c) Conductivity $(\sigma)$ vs time curve corresponding to 3 consecutive vacuum-nitrogen cycles measurements on a $~ 30 \mathrm{~nm}$ Cu-CAT-1 film. d) Normalized average Cu-CAT-1 conductivities represented as $\operatorname{Ln}\left(\sigma / \sigma_{N_{2}}\right)$ measured under different ambient conditions on 12 different $\sim 30 \mathrm{~nm}$ films. $\sigma_{\mathrm{N} 2}$ is the conductivity of the film when exposed to a $\mathrm{N}_{2}$ atmosphere.

of MOFs is measured to ensure meaningful comparison between different materials.

To rationalise the experimental data, we calculated the electronic structure of Cu-CAT-1 in vacuum and in the presence of different guests by using dispersion-corrected density functional theory (DFT-D3) and the reported structural model for the MOF, ${ }^{[23]}$ that fixes a slipped-parallel (AB) stacking arrangement with an interlayer separation of $3.3 \AA$ (See Figures SI7 and SI8 and computational details in the supplementary information). In absence of guest interactions, Cu-CAT-1 is a semiconductor with a band gap of $0.33 \mathrm{eV}$ (Figure 3a), consistent with the experimental value reported of $0.48 \mathrm{eV}^{[23]}$ The projection of the DOS on individual atoms reveals that near the Fermi level, the major contribution corresponds to the $2 p$ orbitals from $\mathrm{O}$ and $\mathrm{C}$ atoms in the linker, indicating strong $\pi-\pi$ interactions between the stacking layers. This suggests that interlayer interactions might be quite effective in controlling the electronic properties of this material, as previously suggested for the isostructural $\mathrm{Ni}_{3}(\mathrm{HITP})_{2}{ }^{[32,33]}$ To corroborate this point, we calculated the electronic structure of the solid by imposing different gallery heights up to $5.36 \AA$. As shown in Figure SI9, increasing the interlayer separation triggers an acute increase in the band gap up to $0.58 \mathrm{eV}$. This suggests a dominant role of the electronic overlap between neighbouring layers in the electrical properties of this family of materials. To investigate the effect of host/guest interactions, we optimized the structure of Cu-CAT-1 in presence of $\mathrm{N}_{2}, \mathrm{H}_{2} \mathrm{O}$ and $\mathrm{NH}_{3}$. The relative humidity at room conditions in our laboratory is $65 \%$, so we assumed that modelling environmental conditions as $\mathrm{H}_{2} \mathrm{O}$ was a fair assumption to simplify calculations. As shown in Figure $\mathbf{3 b}$, the interaction of $\mathrm{N}_{2}$ molecules with $\mathrm{Cu}^{\prime \prime}$ centers is extremely weak with $\mathrm{Cu}-\mathrm{N}$ 
a)

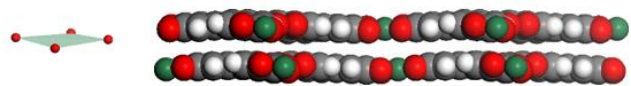

Int. separation $=3.3 \AA$

b)

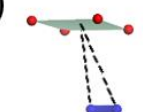

Cu-X $=4.3 \AA$

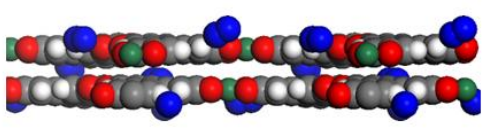

Int. separation $=3.3 \AA$

c)

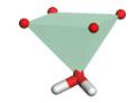

$\mathrm{Cu}-\mathrm{X}=2.7 \AA$

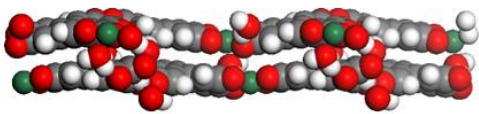

Int. separation $=3.7 \AA$

d)

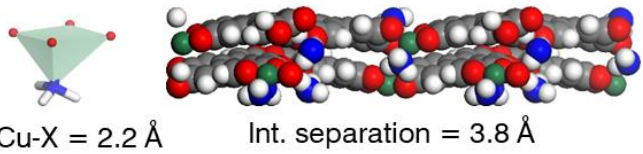

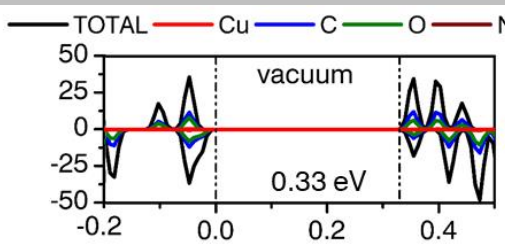

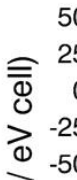

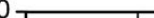

,
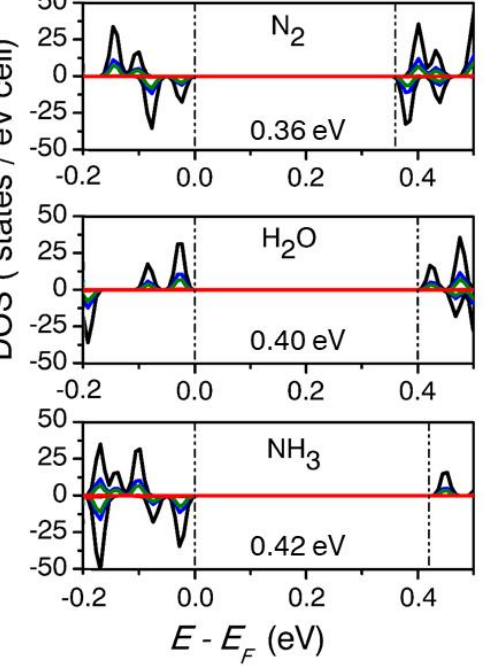

thickness (10-50 nm) displays a linear dependence at the hightemperature regime, consistent with a thermally activated mechanism for charge transport. ${ }^{[23]}$ Provided this mechanism is respected, this shall enable direct comparison of conductivity values with the changes on the electronic structure of the solid upon guest loading (In $\square$ a Eg). ${ }^{[21]}$ Figure $4 a$ shows that the experimental variations in the conductivity of Cu-CAT-1 under the different atmosphere environments studied follow a linear correlation with the calculated band gap values. In order to directly observe the variation of the electronic properties of Cu-CAT-1 upon interaction with gases, we performed macroscopic Kelvin Probe measurements before and after exposure to $\mathrm{NH}_{3}$ of equivalent Cu-CAT-1 thin films deposited onto Glass/Au substrates functionalized with a 1-dodecanethiol (C12S) SAM. As shown in Figure 4b and Sl10, we observed a large decrease of the contact potential difference (CPD) from $312 \pm 4 \mathrm{mV}$ for the pristine Cu-CAT- 1 film to $158 \pm$

distances close to $4.3 \AA$ A. Still, guest infiltration slightly distorts the internal structure of the layers compared to the optimized equilibrium structure in vacuum, for a slight increase in the band gap of $0.03 \mathrm{eV}$. In turn, our calculations suggest a stronger interaction of $\mathrm{H}_{2} \mathrm{O}$ and $\mathrm{NH}_{3}$ molecules with open metal sites. Guest coordination disrupts the square planar geometry of the $\mathrm{Cu}^{\prime \prime}$ centers into a distorted trigonal prism featuring $\mathrm{Cu}-\mathrm{X}$ distances of $2.7 \AA$ and $2.2 \AA$, respectively (Figure 3c,d). This structural change is also linked to a slight variation of the unit cell parameters. Whilst $\mathrm{a}$ and $\mathrm{b}$ axis remain almost constant, $\mathrm{c}$ undergoes an expansion of close to $3 \%$ (Table SI2). These changes to the interlayer separation are quite small. The changes to the internal structure of the layer caused by direct interaction of the guest with the open metal sites possibly have a stronger impact over the electronic structure, for band gaps of $0.40 \mathrm{eV}$ and $0.42 \mathrm{eV}$ after $\mathrm{H}_{2} \mathrm{O}$ and $\mathrm{NH}_{3}$ infiltration. We previously confirmed that the experimental conductivity of $\mathrm{Cu}-\mathrm{CAT}-1$ films with variable

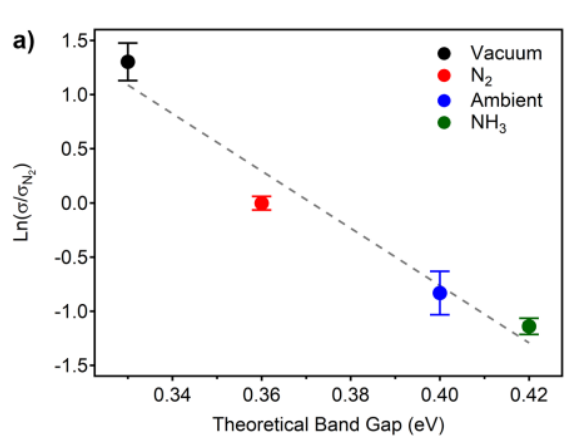

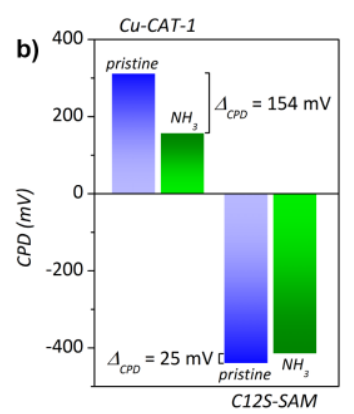

$12 \mathrm{mV}$ upon exposure to $\mathrm{NH}_{3}$ vapours for 5 minutes. This translates in a work function difference of $0.15 \mathrm{eV}\left(\Delta_{\mathrm{CPD}}=154\right.$ $\mathrm{mV}$ ), same order of magnitude than the electronic changes predicted by DFT-calculations. As a reference, we performed the same experiment on a Glass/Au/C12S substrate. In this case, we observed an initial CPD of $-440 \pm 2 \mathrm{mV}$, which is essentially unvaried when exposed to $\mathrm{NH}_{3}(-415 \pm 3 \mathrm{mV})$. Moreover, we performed infrared reflection absorption spectroscopy (IRRAS) of the above Glass/Au/C12S/Cu-CAT-1 films. Figure $4 \mathrm{c}$ shows the IRRAS spectra before and after exposure to $\mathrm{NH}_{3}$, the appearance of $\mathrm{NH}_{3}$ stretching bands at 3336,3265 and $3170 \mathrm{~cm}^{-1}$ and a strong bending peak at $1620 \mathrm{~cm}^{-1}$, due to the coordination of $\mathrm{NH}_{3}$ to $\mathrm{Cu}^{1 !},{ }^{[34]}$ further demonstrates the strong interaction of $\mathrm{NH}_{3}$ with $\mathrm{Cu}$ CAT-1 thin films. Powder $x$-Ray diffraction of the bulk microcrystalline solid also confirm reversible changes in the interlayer separation of Cu-CAT-1 after exposure to $\mathrm{NH}_{3}$ vapours, consistent with the DFT model (Figure Sl11)

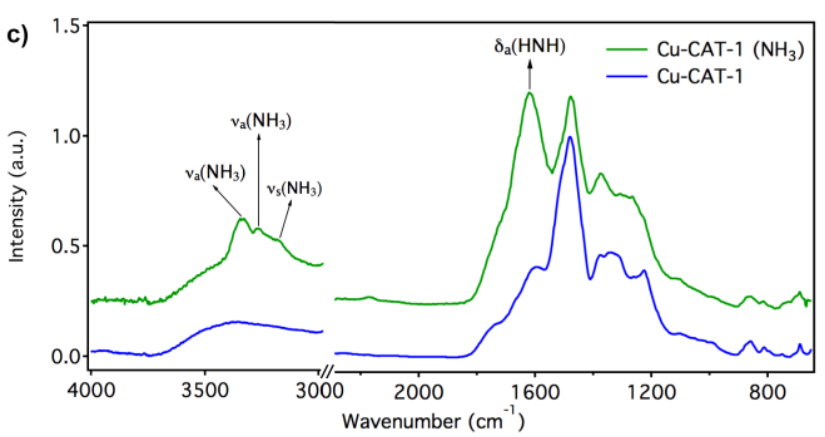

Figure 4. a) Average and standard deviations of $L n\left(\sigma / \sigma_{\mathrm{N} 2}\right)$ values measured in $\sim 30 \mathrm{~nm}$ Cu-CAT-1 films compared with the theoretical bandgap calculated for each gas environment. The grey dotted line is a linear fit of the data. b) Kelvin probe measurements of a Glass/Au/C12S/Cu-CAT-1 film (top) and a reference Glass/Au/C12S substrate (bottom), before and after exposure to $\mathrm{NH}_{3}$ vapours. c) IRRAS spectra of Cu-CAT-1 film before and after exposure to $\mathrm{NH}_{3}$ vapours. Abbreviations: $\mathrm{V}_{\mathrm{a}}$, asymmetric stretching; $v_{s}$, symmetric stretching; $\delta_{a}$, asymmetric bending. 
In summary, our results indicate that the origin of the chemiresistive response of this family of conductive MOFs is linked to the direct interaction of gas molecules with the $\mathrm{Cu}^{\text {Il }}$ sites. This results in slight distortions of the internal structure of the layer or more acute changes in the coordination geometry of the metal node for concomitant modifications of the band gap of the solid. Our findings are also consistent with previous reports, ${ }^{[27-29]}$ that suggest a possible relationship between the nature of the metal nodes and the coordination ability of the analytes with the intensity and selectivity of the "turn-on" response. We are confident this information will be of use to help guiding the design of advanced sensory platforms based on the rational optimization of the chemical functionality and electronic structure of MOFs.

\section{Acknowledgements}

This work was supported by the EU (ERC Stg Chem-fs-MOF 714122, SPIN2D H2020/2014-659378), the Spanish MINECO (MDM-2015-0538, RYC-2012-10894) and BSC-RES computational resources. V.R.-G. and G.E-A. thank the Spanish MECD and GV for FPU13/03203 and Santiago Grisolía predoctoral grants. We thank A. López-Muñoz, F. González, J. Heredero Calero, D. Vie and J. Castells-Gil for their technical support.

Keywords: Metal-Organic Framework • ultrathin film • electrical conductivity $\bullet$ chemiresistive sensing $\bullet$ device

[1] I. Stassen, N. Burtch, A. Talin, P. Falcaro, M. Allendorf, R. Ameloot, Chem. Soc. Rev. 2017, 46, 3185-3241.

[2] D. Feng, T. Lei, M. R. Lukatskaya, J. Park, Z. Huang, M. Lee, L. Shaw, S. Chen, A. A. Yakovenko, A. Kulkarni, et al., Nature Energy 2018, 3, 30-36

[3] W.-H. Li, K. Ding, H.-R. Tian, M.-S. Yao, B. Nath, W.-H. Deng, Y Wang, G. Xu, Adv. Func. Mater. 2017, 27, 1702067.

[4] D. Sheberla, J. C. Bachman, J. S. Elias, C.-J. Sun, Y. Shao-Horn, M. Dincă, Nat. Mater. 2017, 16, 220-224.

[5] G. Wu, J. Huang, Y. Zang, J. He, G. Xu, J. Am. Chem. Soc 2017, 139, 1360-1363.

[6] N. Lahiri, N. Lotfizadeh, R. Tsuchikawa, V. V. Deshpande, J. Louie, J. Am. Chem. Soc. 2017, 139, 19-22.

[7] X. Huang, P. Sheng, Z. Tu, F. Zhang, J. Wang, H. Geng, Y. Zou,

C.-A. Di, Y. Yi, Y. Sun, et al., Nat. Commun. 2015, 6, 7408

[8] B. Bhattacharya, A. Layek, M. Mehboob Alam, D. K. Maity, S

Chakrabarti, P. P. Ray, D. Ghoshal, Chem. Commun. 2014, 50, 7858-7861.

[9] L. Sun, B. Liao, D. Sheberla, D. Kraemer, J. Zhou, E. A. Stach, D. Zakharov, V. Stavila, A. A. Talin, Y. Ge, et al., Joule 2017, 1, 168-177.

[10] K. J. Erickson, F. Léonard, V. Stavila, M. E. Foster, C. D. Spataru, R. E. Jones, B. M. Foley, P. E. Hopkins, M. D. Allendorf A. A. Talin, Adv. Mater. 2015, 27, 3453-3459.

[11] Z. Wang, D. Nminibapiel, P. Shrestha, J. Liu, W. Guo, P. G Weidler, H. Baumgart, C. Wöll, E. Redel, ChemNanoMat 2016, 2, 67-73.

[12] S. Rana, R. Rajendra, B. Dhara, P. K. Jha, N. Ballav, Adv. Mater Interfaces 2016, 3, 1500738 .

[13] L. Mendecki, K. A. Mirica, ACS Appl. Mater. Interfaces 2018, 10, 19248-19257.

[14] F. Gándara, F. J. Uribe-Romo, D. K. Britt, H. Furukawa, L. Lei, R Cheng, X. Duan, M. O'Keeffe, O. M. Yaghi, Chem-Eur J. 2012, 18, 10595-10601.

[15] M. Hmadeh, Z. Lu, Z. Liu, F. Gándara, H. Furukawa, S. Wan, V. Augustyn, R. Chang, L. Liao, F. Zhou, et al., Chem. Mater. 2012, 24, 3511-3513.

[16] L. Sun, C. H. Hendon, M. A. Minier, A. Walsh, M. Dincă, J. Am Chem. Soc. 2015, 137, 6164-6167.

L. Sun, C. H. Hendon, S. S. Park, Y. Tulchinsky, R. Wan, F. Wang, A. Walsh, M. Dincă, Chem. Sci. 2017, 43, 5994.

E. A. Dolgopolova, A. J. Brandt, O. A. Ejegbavwo, A. S. Duke, T. D. Maddumapatabandi, R. P. Galhenage, B. W. Larson, O. G. Reid, S. C. Ammal, A. Heyden, et al., J. Am. Chem. Soc. 2017, 139, 5201-5209.
[19] A. A. Talin, A. Centrone, A. C. Ford, M. E. Foster, V. Stavila, P. Haney, R. A. Kinney, V. Szalai, F. El Gabaly, H. P. Yoon, et al., Science 2014, 343, 66-69.

[20] I. Hod, O. K. Farha, J. T. Hupp, Chem. Commun. 2016, 52 1705-1708.

[21] L. Sun, M. G. Campbell, M. Dincă, Angew. Chem. Int. Ed. 2016, $55,3566-3579$.

[22] M. Ko, L. Mendecki, K. A. Mirica, Chem. Commun. 2018, 9, 9451

[23] V. Rubio-Giménez, M. Galbiati, J. Castells-Gil, N. Almora Barrios, J. Navarro-Sánchez, G. Escorcia Ariza, M. Mattera, T. Arnold, J. Rawle, S. Tatay, et al., Adv. Mater. 2018, 55, 1704291.

[24] T. Kambe, R. Sakamoto, K. Hoshiko, K. Takada, M. Miyachi, J. H. Ryu, S. Sasaki, J. Kim, K. Nakazato, M. Takata, et al., J. Am. Chem. Soc. 2013, 135, 2462-2465.

[25] D. Sheberla, L. Sun, M. A. Blood-Forsythe, S. Er, C. R. Wade, C K. Brozek, A. Aspuru-Guzik, M. Dincă, J. Am. Chem. Soc. 2014, 136, 8859-8862.

[26] J. Cui, Z. Xu, Chem. Commun. 2014, 50, 3986-3988.

[27] M. G. Campbell, D. Sheberla, S. F. Liu, T. M. Swager, M. Dincă, Angew. Chem. Int. Ed. 2015, 54, 4349-4352.

[28] M. G. Campbell, S. F. Liu, T. M. Swager, M. Dincă, J. Am. Chem Soc. 2015, 137, 13780-13783.

[29] M.-S. Yao, X.-J. Lv, Z.-H. Fu, W.-H. Li, W.-H. Deng, G.-D. Wu, G. Xu, Angew. Chem. Int. Ed. 2017, 56, 16510-16514.

[30] V. Rubio-Giménez, S. Tatay, F. Volatron, F. J. Martínez-Casado, C. Martí-Gastaldo, E. Coronado, J. Am. Chem. Soc. 2016, 138, 2576-2584.

[31] L. Sun, S. S. Park, D. Sheberla, M. Dincă, J. Am. Chem. Soc. 2016, 138, 14772-14782.

[32] M. E. Foster, K. Sohlberg, C. D. Spataru, M. D. Allendorf, J. Phys. Chem. C 2016, 120, 15001-15008.

[33] M. E. Foster, K. Sohlberg, M. D. Allendorf, A. A. Talin, J. Phys. Chem. Lett. 2018, 9, 481-486.

[34] K. Nakamoto, Infrared and Raman Spectra of Inorganic and Coordination Compounds, John Wiley \& Sons, Inc., Hoboken, NJ, USA, 2008. 


\section{COMMUNICATION}

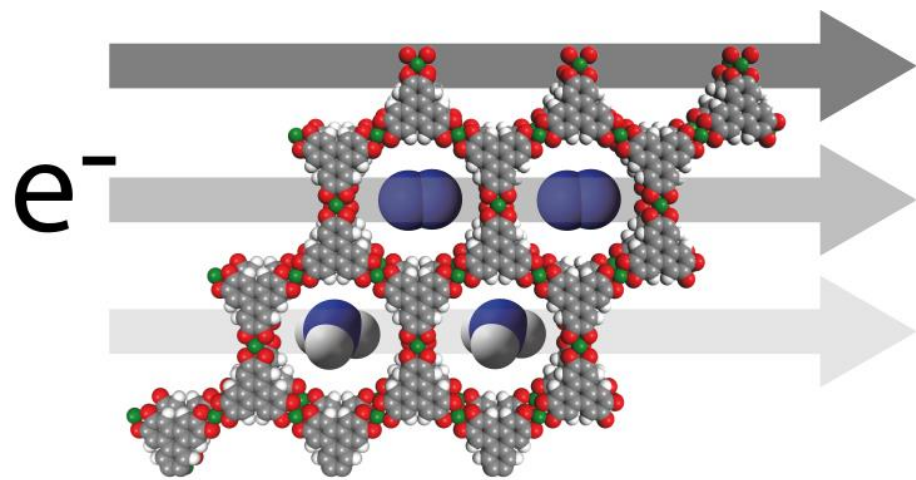

Víctor Rubio-Giménez, ${ }^{\dagger}$ Neyvis AlmoraBarrios, ${ }^{\dagger}$ Garin Escorcia-Ariza, ${ }^{\dagger}$ Marta Galbiati, Michele Sessolo Sergio Tatay* and Carlos Martí-Gastaldo*

Origin of the Chemiresistive Response of Ultrathin Films of Conductive Metal-Organic Frameworks

Electrical response to atmospheric guests: By combining experimental data with computational modelling, we describe a possible origin for the underlying mechanism of the direct dependence of the electrical response with MOF/guest interaction phenomenon in ultrathin films $(\sim 30 \mathrm{~nm})$ of Cu-CAT-1. 